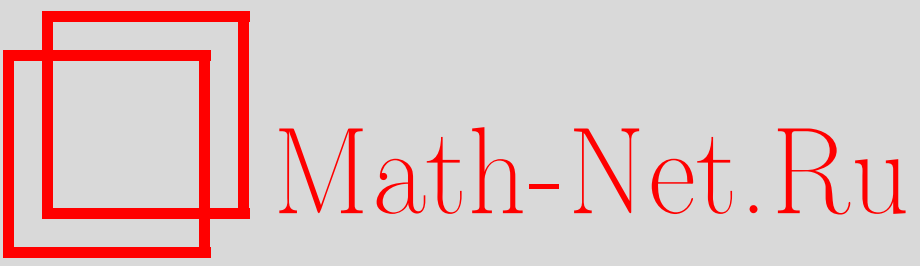

В. Р. Халилов, Фермионы в сильных внешних полях в $2+1$ и $1+1$ измерениях, TMФ, 2000, том 122, номер 3, 372-384

DOI: https://doi.org/10.4213/tmf573

Использование Общероссийского математического портала Math-Net.Ru подразумевает, что вы прочитали и согласны с пользовательским соглашением

http://www . mathnet.ru/rus/agreement

Параметры загрузки:

IP: 54.147 .182 .235

26 апреля 2023 г., 15:26:59 


\author{
ТЕОРЕТИЧЕСКАЯ \\ И МАТЕМАТИЧЕСКАЯ \\ ФИЗИКА \\ Том 122, № 3 \\ март, 2000
}

(C) 2000 г.

В.Р. Халилов*

\title{
ФЕРМИОНЫ В СИЛЬНЫХ ВНЕШНИХ ПОЛЯХ В $2+1$ И $1+1$ ИЗМЕРЕНИЯХ
}

\begin{abstract}
Эффект образования электрон-позитронных пар из вакуума внешним кулоновским полем изучается в $(2+1)$-мерной квантовой электродинамике. Показано, что если электромагнитная константа связи $Z \alpha$ превышает значение $0.62(Z=85)$, то для простой модели протяженного источника (ядра) низший электронный уровень энергии пересекает границу нижнего континуума (дираковского моря) и в нижнем континууме появляется дырка (положительно заряженный фермион-позитрон). Получено уравнение, определяющее уровни энергии основного и возбужденных электронных состояний вблизи границы нижнего континуума в сильном кулоновском поле ядра. Критический заряд ядра найден для нескольких низших электронных состояний. Показано, что величина критического заряда в $2+1$ измерениях существенно меньше его значения для той же модели в $3+1$ измерениях. Изучено влияние внешнего сверхсильного постоянного магнитного поля на спектр энергий электрона в кулоновском поле и величину критического заряда ядра в $3+1$ измерениях. Проблема сведена к решению задачи в ограниченном кулоновском поле в $1+1$ измерениях, но без магнитного поля. Рассмотрено взаимодействие фермиона со скалярным внешним полем в $2+1$ и $1+1$ измерениях.
\end{abstract}

\section{1. ВВЕДЕНИЕ}

В последнее время значительно возрос интерес к изучению поведения квантовых систем фермионов в присутствии интенсивных внешних полей в пространствах пониженных размерностей. Этот интерес можно связать с двумя причинами. Так, например, ряд эффектов в конденсированных средах указывает на сушествование квантовых систем со спектром энергий, определяемым гамильтонианом уравнения Дирака в $2+1$ измерениях $[1,2]$. Другой пример связан с поведением электронов в сверхсильном магнитном поле. В частности, можно показать, что в сверхсильном однородном магнитном поле напряженности $B>B_{\text {cr }} \equiv m^{2} /|e|$ электрон в перпендикулярной полю плоскости локализован в области с линейным размером порядка так называемой "магнитной длины" $a=\sqrt{2 /|e B|}$, т.е. линейный размер области локализации в этой плоскости оказывается меньше комптоновской длины волны электрона [3]. Такое “вымораживание" двух пространственных степеней свободы фактически позволяет свести ряд задач (3+1)-мерной квантовой электродинамики $\left(\mathrm{K} Э Д_{3+1}\right)$ к задачам $\mathrm{K} Э Д_{1+1}$.

* Московский государственный университет, Москва, Россия. E-mail: khalilov@thc.phys.msu.su 
В связи с этим изучение поведения заряженных квантовых частиш в присутствии сильных внешних полей в области релятивистских энергий в пространствах пониженных размерностей, а также исследование обусловливаемых присутствием сильного поля физических эффектов актуально в настояшее время и с точки зрения возможных практических приложений. Одним из таких эффектов является эффект образования электронпозитронной пары из вакуума кулоновским полем, который для случая кулоновского поля протяженного источника в трех пространственных измерениях был предсказан в статье [4] и затем исследован во многих работах [5-11]. Точные решения уравнения Дирака в сильном кулоновском поле для электрона обсуждались недавно в работах [12].

В данный работе рассматривается эффект рождения электрон-позитронных пар из вакуума в КЭД $2+1$ и КЭД $1+1$ сильным кулоновским полем. Исследуются решения уравнения Дирака и спектр энергий электронных состояний в сильном кулоновском поле протяженного ядра вблизи границы нижнего континуума, а также изучается вопрос о влиянии внешнего сверхсильного постоянного однородного магнитного поля на спектр энергий электрона в кулоновском поле, на критический заряд и на вероятность рождения электрон-позитронной пары из вакуума сильным кулоновским полем в $3+1$ измерениях. Рождение позитронов сверхкритическим кулоновским полем рассмотрено в предположении, что в нижний континуум погружаются несколько связанных состояний.

Кроме взаимодействия фермиона с внешним (векторным) электромагнитным полем, рассматривается модель, в которой массивные фермионы взаимодействуют с внешним скалярным полем. В трех пространственных измерениях взаимодействие массивного дираковского поля со скалярным внешним полем, как полагают [11], моделирует самосогласованное поле кварковой системы. Ниже найдены решения и спектры энергий уравнения Дирака во внешнем скалярном поле кулоновского типа $U(r)=-q / r$ в $2+1$ измерениях, а также в скалярном внешнем поле $U(x)=-q /(|x|+a)$ в $1+1$ измерениях. Модель с таким взаимодействием в $1+1$ измерениях интересна потому, что она приводит к существованию фермионного состояния с нулевой энергией, значение которого кратко обсуждается.

В статье используется система единиц, в которой $c=\hbar=1$.

\section{2. СПЕКТР ЭНЕРГИЙ ЭЛЕКТРОНА В КУЛОНОВСКОМ ПОЛЕ В $2+1$ ИЗМЕРЕНИЯХ}

Поскольку [2] в $2+1$ измерениях алгебра матриц Дирака может быть выражена через матрицы Паули как $\gamma^{0}=\sigma_{3}, \gamma^{k}=i \sigma_{k}$, уравнение Дирака для электрона массы $m$ и заряда $e=-e_{0}, e_{0}>0$, взаимодействие которого с внешним полем удовлетворяет принципу “минимальности” в двух пространственных измерениях, можно записать в виде

$$
\left(i \partial_{t}-H_{\mathrm{D}}\right) \Psi=0
$$

где

$$
H_{\mathrm{D}}=\boldsymbol{\alpha} \mathbf{P}+\beta m+e A^{0} \equiv \sigma_{1} P_{2}-\sigma_{2} P_{1}+\sigma_{3} m+e A^{0}
$$


- гамильтониан, $P_{\mu}=i \partial_{\mu}-e A_{\mu}$ - оператор обобшенного импульса электрона, $A_{\mu}$ - вектор-потенциал внешнего электромагнитного поля, а полный момент импульса электрона имеет только одну компоненту $J_{z}=L_{z}+S_{z}$, где $L_{z}=-i \partial / \partial \varphi$ и $S_{z}=\sigma_{3} / 2$.

Решение уравнения Дирака во внешнем кулоновском поле, описываемом вектором-потенциалом

$$
A^{0}(r)=-\frac{Z e_{0}}{r}, \quad A^{x}=A^{y}=0,
$$

удобно искать в полярных координатах $r, \varphi$. Положим

$$
\Psi(t, \mathbf{x})=\frac{1}{\sqrt{2 \pi}} \exp (-i E t+i l \varphi) \psi(r, \varphi)
$$

где $E$ - энергия, $l$ - целое число и

$$
\psi(r, \varphi)=\left(\begin{array}{c}
f(r) \\
g(r) e^{i \varphi}
\end{array}\right)
$$

Заметим, что волновая функция (4) является собственной функцией операторов Гамильтона $H_{\mathrm{D}}$ и полного момента $J_{z}$ с собственными значениями $E$ и $l+1 / 2$ соответственно.

Подставляя соотношения (4) и (5) в уравнение (1) и учитывая равенства

$$
P_{x} \pm i P_{y}=-i e^{ \pm i \varphi}\left(\frac{\partial}{\partial r} \pm \frac{i}{r} \frac{\partial}{\partial \varphi}\right)
$$

получим следующую систему уравнений:

$$
\begin{array}{r}
\frac{d f}{d r}-\frac{l}{r} f+\left(E+m+\frac{Z \alpha}{r}\right) g=0, \\
\frac{d g}{d r}+\frac{1+l}{r} g-\left(E-m+\frac{Z \alpha}{r}\right) f=0,
\end{array}
$$

где $\alpha \equiv e^{2}=1 / 137$ - постоянная тонкой структуры.

В кулоновском поле точечного ядра решения и дискретный спектр энергий $E<m$ уравнения Дирака были найдены в работах [12]. В частности, дискретный спектр энергий электрона в поле (3) имеет вид

$$
E=m\left[1+\frac{(Z \alpha)^{2}}{\left(n_{r}+\sqrt{\left(l+\frac{1}{2}\right)^{2}-(Z \alpha)^{2}}\right)^{2}}\right]^{-1 / 2}
$$

причем оказывается, что допустимы следуюшие значения квантового числа $n_{r}$ : $0,1,2, \ldots$ при $l \geqslant 0$ и $1,2,3, \ldots$ при $l<0$.

Энергия электрона на низшем уровне $\left(l=n_{r}=0\right)$

$$
E_{0}=m \sqrt{1-(2 Z \alpha)^{2}}
$$


и обращается в нуль при $Z \alpha=1 / 2$. Заметим, что т.к. рассматриваемое здесь кулоновское поле притягивает электроны и отталкивает позитроны, то спектры энергий для частиц (электронов) и античастиц (позитронов) несимметричны.

Спектры энергий для частиц и античастиц будут симметричны в случае взаимодействия массивных фермионов со скалярным внешним полем кулоновского типа $U(r)=$ $-q / r$. Гамильтониан уравнения Дирака (1) для такого взаимодействия имеет вид

$$
H_{\mathrm{D}}=\boldsymbol{\alpha} \mathbf{p}+\beta(m+U(r)) \equiv \sigma_{1} p_{2}-\sigma_{2} p_{1}+\sigma_{3}(m+U(r)),
$$

где $p_{k}=i \partial_{k}-$ пространственные компоненты оператора импульса. Уравнения движения массивного фермиона, взаимодействуюшего со скалярным внешним полем $U(r)=$ $-q / r$, получаются из (7) при изменении знака перед последним членом во второй строке и замене $Z \alpha$ на $q$. Мы приведем здесь только формулу для спектра энергий фермиона во внешнем скалярном поле

$$
E_{ \pm}= \pm m\left[1-\frac{q^{2}}{\left(n_{r}+\sqrt{(l+1 / 2)^{2}+q^{2}}\right)^{2}}\right]^{1 / 2} .
$$

Величина $E_{ \pm}$для $l=n_{r}=0$ лишь стремится к нулю при $q \rightarrow \infty$ :

$$
E_{(0) \pm}= \pm \frac{m}{\sqrt{1+4 q^{2}}} \rightarrow 0
$$

\section{3. ПРОТЯЖЕННЫЙ КУЛОНОВСКИЙ ЦЕНТР. КРИТИЧЕСКИЙ ЗАРЯД}

Энергетический спектр электрона в кулоновском поле ядра с $Z \alpha \geqslant 1 / 2$ имеет физический смысл только с учетом конечных размеров ядра, создаюшего кулоновский потенциал [13], т.е. следует рассматривать потенциал, обрезанный на некотором расстоянии $R$. В случае трех пространственных измерений спектр энергий электрона в сильном поле обрезанного (на малых расстояниях) кулоновского потенциала впервые был исследован в статье [13]. С ростом $Z$ в области $Z>137$ уровни энергии электрона становятся отрицательными и продолжают опускаться до границы нижнего континуума $-m$. Значение $Z=Z_{\mathrm{cr}}$, при котором нижний уровень энергии электрона достигает гранищы нижнего континуума, назьвают критическим для основного состояния [9-11]. Как показано в работах [12], в случае двух пространственных измерений поведение уровней энергии электрона аналогично трехмерному случаю, однако пересечение границы нижнего континуума $-m$ имеет место при значительно меньших значениях $Z$.

Рассмотрим решения и спектр энергий уравнения Дирака в области $2 Z \approx 137$ и определим соответствуюшие значения $Z_{\mathrm{cr}}$. Исключая из системы $(7)$ функцию $g(r)$, получим

$$
\begin{aligned}
\frac{d^{2} f(r)}{d r^{2}} & +\frac{1}{r} \frac{d f(r)}{d r}+\left(E^{2}-m^{2}+\frac{2 E Z \alpha}{r}+\frac{(Z \alpha)^{2}-l^{2}}{r^{2}}\right) f(r)+ \\
& +\frac{Z \alpha}{r^{2}}\left(E+m+\frac{Z \alpha}{r}\right)^{-1}\left(\frac{d f(r)}{d r}-\frac{l}{r} f(r)\right)=0 .
\end{aligned}
$$


Отметим, что последний член этого уравнения пропорционален $\hbar$. Этот член мал при достаточно больших $r$, однако он влияет на асимптотический вид решений при малых $r \sim \hbar / m c$.

Так как для определения $Z_{\text {сr }}$ достаточно рассмотреть область значений энергий вблизи границы нижнего континуума $-m$, то для функции $F(r)=r f(r)$ из уравнения (13) нетрудно получить уравнение при $E \approx-m$ в виде

$$
\frac{d^{2} F(r)}{d r^{2}}+\left(E^{2}-m^{2}+\frac{2 E Z \alpha}{r}+\frac{(Z \alpha)^{2}-l(l+1)}{r^{2}}\right) F(r)=0 .
$$

Решение уравнения (14), убываюшее при $r \rightarrow \infty$, выражается через функцию Уиттекера вида

$$
F(r)=W_{\beta, i \theta}(2 \lambda r)
$$

где

$$
\beta=\frac{E Z \alpha}{\lambda}, \quad \theta=\sqrt{(Z \alpha)^{2}-\left(l+\frac{1}{2}\right)^{2}}, \quad \lambda=\sqrt{m^{2}-E^{2}} .
$$

Решение для $G(r)=r g(r)$ вблизи значения $E=-m$ находится из равенства

$$
G(r)=\frac{1}{Z \alpha}\left((1+l) F-\frac{d F}{d r}\right)
$$

с использованием уравнения Дирака и рекуррентных соотношений для функций Уиттекера.

Уравнение (14) можно интерпретировать как стационарное уравнение Шредингера, в котором $E^{\prime}=\left(E^{2}-m^{2}\right) / 2 m-$ энергия "частицы", а

$$
U_{\mathrm{eff}}(r)=-\frac{E Z \alpha}{m r}-\frac{(Z \alpha)^{2}-l(l+1)}{2 m r^{2}}
$$

- эффективная потенщиальная энергия. Решение уравнения (14) с $E=-m$ можно выразить через функцию Макдональда мнимого индекса [14]

$$
F(r)=\sqrt{r} K_{2 i \theta}(\sqrt{8 m Z \alpha r}) .
$$

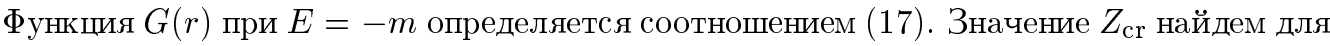
простой модели, задавая потенциал в виде

$$
A_{0}^{Z}(r)= \begin{cases}-\frac{Z e_{0}}{r}, & r \geqslant R \\ -\frac{Z e_{0}}{R}, & r \leqslant R .\end{cases}
$$

В области $r \leqslant R$ уравнение для функции $F(r)$ имеет вид

$$
\frac{d^{2} F}{d r^{2}}-\frac{d F}{r d r}+\left(\left(E+\frac{Z \alpha}{R}\right)^{2}-m^{2}+\frac{1-l^{2}}{r^{2}}\right) F(r)=0 .
$$


Его конечное (при $r=0$ ) решение выражается через функцию Бесселя $J_{n}(z)$ целочисленного индекса $n$ следуюшим образом [14]:

$$
F(r)=r J_{|l|}(\kappa r)
$$

где

$$
\kappa=\sqrt{\left(E+\frac{Z \alpha}{R}\right)^{2}-m^{2}} .
$$

Для нахождения спектра уравнения Дирака необходимо сшить решения в точке $r=R$ :

$$
\left(\frac{G(r)}{F(r)}\right)_{r=R-0}=\left(\frac{G(r)}{F(r)}\right)_{r=R+0} .
$$

В частности, полагая $E=-m$ и учитывая, что параметр $R$ мал по сравнению с комптоновской длиной волны электрона $1 / m$, так что $\kappa \approx Z \alpha / R$, получим трансцендентное уравнение, определяюшее (при фиксированном $R$ ) критический заряд для основного и возбужденных состояний с $l=0$ :

$$
\frac{J_{1}(X)}{J_{0}(X)}=\left(1-\frac{z W_{\beta, i \nu}^{\prime}(x)}{W_{\beta, i \nu}(x)}\right)
$$

или

$$
J_{0}(X)=K_{2 i \nu}(z)
$$

где $X=Z_{\mathrm{cr}} \alpha, \nu=\sqrt{X^{2}-1 / 4}, \beta=-m Z \alpha / \lambda, x=\lambda R, z=\sqrt{8 m R X}$ и штрих означает производную по аргументу функции Уиттекера $x$.

Полученные уравнения удается решить лишь численно. Например, для того чтобы решить уравнение (26), используем представление для функций Макдональда при малых значениях аргумента в виде [12]

$$
\begin{aligned}
K_{\mu}(z)= & \frac{\pi}{2 \sin (\pi \mu)}\left\{\frac{z^{-\mu}}{2^{-\mu} \Gamma(1-\mu)}\left[1+\frac{z^{2}}{4(1-\mu)}+\cdots\right]-\right. \\
& \left.-\frac{z^{\mu}}{2^{\mu} \Gamma(1+\mu)}\left[1+\frac{z^{2}}{4(1+\mu)}+\cdots\right]\right\} .
\end{aligned}
$$

Далее, после несложных преобразований нетрудно получить следующую формулу:

$$
K_{i \nu}(z)=-\left(\frac{\pi}{\nu \operatorname{sh}(\pi \nu)}\right)^{\frac{1}{2}}\left[\left(1+\frac{z^{2}}{4\left(1+\nu^{2}\right)}\right) \sin P-\frac{\nu z^{2}}{4\left(1+\nu^{2}\right)} \cos P\right],
$$

где

$$
P=\nu \ln \frac{|z|}{2}+\arg \Gamma(1-i \nu)
$$

Значения критического заряда $Z_{\mathrm{cr}}$ для состояний с $l=0, n_{r}=1,2,3$ (эти значения получаются при численном решении уравнения (26), в котором функция Макдональда учтена в виде разложения $(28)$ при $R m=0.03)$ примерно равны соответственно 85, 111, 148 . 
Отметим, что $Z_{\text {cr }} \approx 170,230$ при $R m=0.03$ в состояниях $1 s_{1 / 2}, 2 s_{1 / 2}$ для аналогичной модели в трех пространственных измерениях [9-11]. Отметим также, что с уменьшением $R$ уменьшается и $Z_{\text {cr. }}$. Следовательно, вакуум $\mathrm{K} Э Д_{2+1}$ в сильном кулоновском поле должен проявлять неустойчивость по отношению к образованию электрон-позитронных пар при сушественно меньших значениях критического заряда, чем в $\mathrm{K}_{\text {ЭД }}$ 乙 $_{3+1}$.

Получим теперь уравнение, которое определяет спектр энергий электрона вблизи границы нижнего континуума, но для произвольных (целых) значений $l$. Вблизи границы нижнего континуума $E \simeq-m$ при условии $R m \ll 1$ уравнение $(24)$ можно существенно упростить. Для этого используем представление для функций Уиттекера при малых значениях аргумента в виде [14]

$$
W_{\beta, i \theta}(z)=\left\{\frac{\Gamma(2 i \theta)}{\Gamma\left(\frac{1}{2}-\beta+i \theta\right)} z^{\frac{1}{2}-i \theta}+\frac{\Gamma(-2 i \theta)}{\Gamma\left(\frac{1}{2}-\beta-i \theta\right)} z^{\frac{1}{2}+i \theta}\right\}
$$

Далее, после несложных преобразований нетрудно получить формулу

$$
W_{\beta, i \theta}(z)=\frac{|\Gamma(2 i \theta)|}{\left|\Gamma\left(\frac{1}{2}-\beta+i \theta\right)\right|} 2 z^{\frac{1}{2}} \cos (\Phi)
$$

где

$$
\Phi=-\theta \ln (2 \lambda r)+\arg \Gamma(2 i \theta)-\arg \Gamma\left(\frac{1}{2}-\beta+i \theta\right)
$$

Вычислив производную по аргументу $z$ в соотношении (30) и подставляя полученный результат и функцию (30) в равенство (24), получим трансцендентное уравнение, неявно определяющее спектр энергий как для основного, так и для возбужденных состояний с учетом конечности ядра:

$$
\frac{\kappa R J_{l+1}(\kappa R)}{J_{l}(\kappa R)}=l+\frac{1}{2}-\theta \operatorname{tg}(\Phi)
$$

или эквивалентно

$$
\Phi=\operatorname{arctg} \Pi+\pi n_{r}
$$

где

$$
\Pi=-\left(\frac{\kappa R J_{l+1}(\kappa R)}{J_{l}(\kappa R)}-l-\frac{1}{2}\right) \theta^{-1} .
$$

Из уравнения (31) можно также найти критический заряд протяженного ядра при произвольных значениях $l$, причем как для основного, так и для возбужденных состояний. Решение уравнения (31) численно дает $Z_{\mathrm{cr}} \approx 108$ при $R m=0.03$ для состояния $l=-1$, $n_{r}=1$. Отметим, что $Z_{\text {cr }} \approx 185$ при $R m=0.03$ в случае состояния $2 p_{1 / 2}$ для аналогичной модели в КЭД $3+1[11]$. 


\section{4. ВЛИЯНИЕ СВЕРХСИЛЬНОГО МАГНИТНОГО ПОЛЯ НА СПЕКТР ЭНЕРГИЙ ЭЛЕКТРОНА}

В сверхсильном магнитном поле область движения электрона в перпендикулярной полю плоскости имеет линейный размер порядка $a=\sqrt{2 /|e B|}$, а кулоновский потенциал точечного ядра эффективно обрезается в направлении магнитного поля на расстояниях порядка $a[3]$. Это позволяет свести задачу о влиянии внешнего магнитного поля на критический заряд в $3+1$ измерениях к эффективной задаче о спектре энергий электрона в ограниченном кулоновском поле в $1+1$ измерениях. В $1+1$ измерениях имеются только две $\gamma$-матрицы Дирака. Здесь мы будем использовать представление

$$
\gamma^{0}=\sigma_{3}, \quad \gamma^{1}=i \sigma_{1}
$$

(эквивалентно для матриц $\beta, \alpha_{1}: \beta=\sigma_{3}, \alpha_{1}=-\sigma_{2}$ ).

Гамильтониан уравнения Дирака (1) во внешнем кулоновском поле (магнитное поле в $3+1$ измерениях направлено по $x$ ) имеет вид

$$
H_{\mathrm{D}}=\alpha_{1} P_{1}+\beta m+e A^{0} \equiv i \sigma_{2} \partial_{x}+\sigma_{3} m+e A^{0}
$$

Решение уравнения Дирака с гамильтонианом (34) во внешнем поле

$$
A^{0}(x, a)=-\frac{Z e_{0}}{|x|+a}
$$

( $a$ - параметр обрезания) ищем в виде

$$
\Psi(t, x)=\exp (-i E t) \Psi(x)
$$

причем

$$
\Psi(x)=\left(\begin{array}{l}
\psi_{1}(x) \\
\psi_{2}(x)
\end{array}\right)
$$

- двухкомпонентная функция (спинор).

В результате приходим к следующей системе уравнений:

$$
\frac{d \psi_{1}}{d x}-\left(E+m+\frac{Z \alpha}{|x|+a}\right) \psi_{2}=0, \quad \frac{d \psi_{2}}{d x}+\left(E-m+\frac{Z \alpha}{|x|+a}\right) \psi_{1}=0
$$

Решения следует искать отдельно для областей положительных и отрищательных значений $x$.

Введем обозначения $b_{+}=\sqrt{m+E}, b_{-}=\sqrt{m-E}, \lambda=b_{+} b_{-} \equiv \sqrt{m^{2}-E^{2}}$ и перейдем к переменной $z=2 \lambda(|x|+a)$. Вводя функции

$$
\psi_{1}=\frac{u+v}{b_{-}}, \quad \psi_{2}=\frac{u-v}{b_{+}}
$$


после простых преобразований приходим к следующим уравнениям для $u$ и $v$ :

$$
\begin{aligned}
& \frac{d^{2} u}{d z^{2}}+\frac{1}{z} \frac{d u}{d z}+\left(-\frac{1}{4}+\frac{2 E Z \alpha-\lambda}{2 \lambda z}+\frac{(Z \alpha)^{2}}{z^{2}}\right) u=0 \\
& \frac{d^{2} v}{d z^{2}}+\frac{1}{z} \frac{d v}{d z}+\left(-\frac{1}{4}+\frac{2 E Z \alpha+\lambda}{2 \lambda z}+\frac{(Z \alpha)^{2}}{z^{2}}\right) v=0
\end{aligned}
$$

Решения уравнений (40) и (41) в области положительных $x$, убывающие при $x \rightarrow \infty$, выражаются через функции Уиттекера

$$
u=\frac{A W_{\beta-\frac{1}{2}, i \rho}(z)}{\sqrt{z}}, \quad v=\frac{B W_{\beta+\frac{1}{2}, i \rho}(z)}{\sqrt{z}},
$$

здесь

$$
\beta=\frac{E Z \alpha}{\lambda}, \quad \rho=Z \alpha
$$

Решение системы уравнений (38) имеет вид

$$
\begin{aligned}
\psi_{1} & =\frac{A W_{\beta-\frac{1}{2}, i \rho}(z)+B W_{\beta+\frac{1}{2}, i \rho}(z)}{b_{-\sqrt{z}}}, \\
\psi_{2} & =-\frac{A W_{\beta-\frac{1}{2}, i \rho}(z)-B W_{\beta+\frac{1}{2}, i \rho}(z)}{b_{+\sqrt{z}}},
\end{aligned}
$$

а связь между постоянными $A$ и $B$ определяется соотношением $A \lambda=B Z \alpha m$. Волновые функции в области $-\infty<x<0$ получаются аналогично. Решения можно классифицировать по четности: 1 ) четные $\psi_{1}$, нечетные $\left.\psi_{2} ; 2\right)$ четные $\psi_{2}$, нечетные $\psi_{1}$.

Уравнения для определения спектра энергий фермиона в поле (35), очевидно, можно получить из условий $\psi_{2}(0)=0$ для случая 1 и $\psi_{1}(0)=0$ для случая 2. При малых $a m \ll 1$ с учетом разложения функций Уиттекера вблизи нуля $(29)$ и формулы $(30)$ нетрудно получить трансцендентное уравнение, неявно определяюшее спектр энергий фермиона в виде

$$
\rho \ln (2 \lambda a)+\arg \Gamma(-\beta+i \rho)-\frac{1}{2} \operatorname{arctg} \frac{\rho}{\beta}-\arg \Gamma(2 i \rho)=\pi\left(n+\frac{1}{2}\right),
$$

где $n$ - целое число, нумеруюшее уровни энергии. Низшему уровню энергии соответствует значение $n=-1$.

Критический заряд сушественно зависит от параметра $a$, т.е. от величины магнитного поля. При $a m \ll 1$, что соответствует сверхсильным магнитным полям $B \gg B_{\mathrm{cr}}$, величина критического заряда сушественно меньше его значения в отсутствие магнитного поля. Так, решив уравнение (45) численно для $a m=0.1\left(B \sim 10^{2} B_{\mathrm{cr}}\right)$, мы полу-

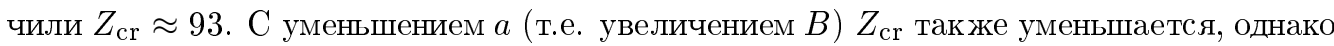
минимальное значение параметра $a$, разумеется, ограничено величиной радиуса реального ядра. 
Любое из уравнений (40) и (41) можно записать и затем интерпретировать как стационарное одномерное уравнение Шредингера. Например, вводя функцию

$$
u(z)=\frac{F(z)}{\sqrt{z}}
$$

мы легко придем от уравнения (40) к уравнению (для $x>0$ )

$$
\frac{1}{2 m} \frac{d^{2} F}{d X^{2}}+\left(\frac{E^{2}-m^{2}}{2 m}+\frac{2 Z \alpha E-\lambda}{2 m X}+\frac{(2 Z \alpha)^{2}+1}{8 m X^{2}}\right) F=0,
$$

где $X=x+a$.

Уравнения (32) и (45) при $Z<Z_{\text {cr }}$ определяют неявно спектр энергий связанных электронных состояний с $m>E>-m$. Можно показать, что решения этих уравне-

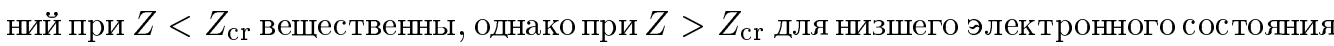
появляется формальное комплексное решение $E=E_{0}-i w$, в котором

$$
E_{0}=-m-c_{1} \alpha\left(Z-Z_{\mathrm{cr}}\right)
$$

( $c_{1}$ - число порядка единицы), а мнимая часть (при $Z-Z_{\text {cr }} \ll Z_{\text {сr }}$ ) экспоненциально мала. Такое решение можно найти из формул (32) и (45), выполняя аналитическое продолжение $E$ как функции от $Z$ в $(32)$ и $(45)$ в область $Z>Z_{\text {cr }}$. Однако при $Z-Z_{\text {cr }} \ll Z_{\text {cr }}$ величину мнимой части с точностью до предэкспоненциального множителя проще оценить другим способом. А именно появление мнимой части означает, что при $Z>Z_{\text {cr }}$ соответствуюшее уравнение Дирака имеет лишь формальное решение с $E=E_{0}-i w$ для

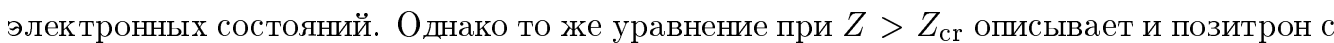
энергией $E=m+c_{1} \alpha\left(Z-Z_{\mathrm{cr}}\right)$. Очевидно, что уравнение Дирака, описывающее позитронные состояния при $Z>Z_{\mathrm{cr}}$, можно получить из уравнения Дирака для электронных состояний, заменив в нем $E$ на $-E$ и $Z$ на $-Z$. Поэтому позитронные состояния при $Z>Z_{\text {сr }}$ являются квазистационарными.

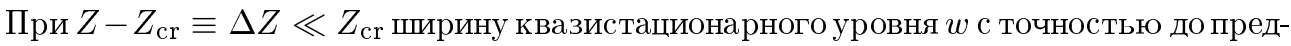
экспоненциального множителя можно оценить в квазиклассическом приближении, вычисляя коэффициент прохождения через потенциальный барьер в уравнении (14) (для случая чисто кулоновского поля в $2+1$ измерениях) или в уравнении (46) (для случая внешних сверхсильного магнитного и кулоновского полей в $3+1$ измерениях). Отметим, что ширина $w$ равна половине обратного времени жизни позитрона в потенциальном барьере или удвоенной вероятности рождения пары кулоновским полем. При $\Delta Z \ll Z_{\text {сr }}$ рождаемые полем позитроны очень медленные и кулоновский барьер для таких позитронов малопроницаем, вследствие чего вероятность рождения пары экспоненциально мала, т.е.

$$
w \sim m \exp \left(-2 \pi Z \alpha\left(\frac{m}{\sqrt{E^{2}-m^{2}}}-1\right)\right) \sim m \exp \left(-c_{2} \sqrt{\frac{Z_{\mathrm{cr}}}{\Delta Z}}\right),
$$

где $c_{2}$ - численньй множитель порядка единицы. Следует подчеркнуть, что экспоненциальная малость вероятности рождения пары имеет место только при $\Delta Z \ll Z_{\text {cr }}$. 


\section{5. О РЕШЕНИИ С НУЛЕВОЙ ЭНЕРГИЕЙ}

Спектры энергий для частиц и античастиц будут симметричны для случая взаимодействия массивных фермионов со скалярным внешним полем $U(x)=-q /(|x|+a)$. Заметим, что теперь параметр $a$, хотя и является параметром обрезания, но не имеет смысла “магнитной длины”. Соответствуюшие уравнения движения можно получить из системы (38) при изменении знака перед последним членом во втором уравнении этой системы и замены $Z \alpha$ на $q$. Однако решения уравнения Дирака в таком поле можно найти несколько проще, если использовать представление

$$
\gamma^{0}=\beta=\sigma_{1}, \quad \gamma^{1}=i \sigma_{3}, \quad \alpha_{1}=\sigma_{2}
$$

В этом представлении стационарное уравнение Дирака во внешнем скалярном поле $U(x)$ имеет вид

$$
\left(-i \sigma_{2} \frac{d}{d x}+\sigma_{1}(m+U(x))\right)\left(\begin{array}{l}
\psi_{1}(x) \\
\psi_{2}(x)
\end{array}\right)=E\left(\begin{array}{l}
\psi_{1}(x) \\
\psi_{2}(x)
\end{array}\right) .
$$

Модель с таким взаимодействием представляет интерес прежде всего потому, что она приводит к сушествованию фермионного состояния с нулевой энергией. Покажем, что уравнение Дирака

$$
\frac{d \psi_{1}}{d x}+\left(m-\frac{q}{|x|+a}\right) \psi_{1}=E \psi_{2}, \quad-\frac{d \psi_{2}}{d x}+\left(m-\frac{q}{|x|+a}\right) \psi_{2}=E \psi_{1}
$$

имеет решение с нулевой энергией.

Связанные уравнения (49) легко привести к паре одномерных уравнений Шредингера для функций $\psi_{1}$ и $\psi_{2}$ :

$$
\begin{aligned}
& \frac{d^{2} \psi_{1}}{d z^{2}}+\left(-\frac{1}{4}+\frac{q m}{\lambda z}+\frac{q(1-q)}{z^{2}}\right) \psi_{1}=0 \\
& \frac{d^{2} \psi_{2}}{d z^{2}}+\left(-\frac{1}{4}+\frac{q m}{\lambda z}-\frac{q(1+q)}{z^{2}}\right) \psi_{2}=0
\end{aligned}
$$

Решения уравнений (50) и (51) в области положительных $x$, убывающие при $x \rightarrow \infty$, выражаются через функции Уиттекера

$$
\psi_{1}(x)=A W_{g, q-1 / 2}(z), \quad \psi_{2}(x)=B W_{g, q+1 / 2}(z)
$$

c $g=q m / \lambda$.

Связь между постоянными $A$ и $B$ можно найти, подставляя (52) в (49) и используя рекуррентные соотношения для функций Уиттекера. В результате мы найдем, что $A(m-$ $\lambda)=B E$. Волновые функции в области $-\infty<x<0$ можно получить, заметив, что уравнения (49) инвариантны относительно преобразований $x \rightarrow-x, \psi_{1}(x) \rightarrow \psi_{1}(-x)$, $\psi_{2}(x) \rightarrow-\psi_{2}(-x), E \rightarrow-E$ или преобразований $x \rightarrow-x, \psi_{1}(x) \rightarrow-\psi_{1}(-x), \psi_{2}(x) \rightarrow$ 
$\psi_{2}(-x), E \rightarrow-E$. Решения в полной области $-\infty<x<\infty$ можно классифицировать по четности: 1 ) четные $\psi_{1}$ и нечетные $\left.\psi_{2}, 2\right)$ четные $\psi_{2}$ и нечетные $\psi_{1}$.

У равнения для определения спектра энергий фермиона в рассматриваемом внешнем скалярном поле, как и выше, можно получить из условия $\psi_{2}(0)=0$ для случая 1 и условия $\psi_{1}(0)=0$ для случая 2 . Нетрудно показать, что в любом из этих случаев спектр энергий фермиона определяется уравнением

$$
q-\frac{q m}{\lambda}=-n
$$

Следовательно, дискретный спектр энергий фермиона во внешнем скалярном поле $U(x)=-q /(|x|+a)$ имеет вид

$$
E_{n}^{2}=m^{2}\left[1-\frac{q^{2}}{(q+n)^{2}}\right]
$$

а соответствуюшее решение является изолированным. Энергия основного уровня $E_{0}=0$ как для частищы, так и для античастищы.

Для решения с нулевой энергией $B=0$, а функция $\Psi_{0}(x)$ имеет вид (при положительных $x$ )

$$
\Psi_{0}(x)=W_{q, q-1 / 2}(2 m(x+a))\left(\begin{array}{l}
1 \\
0
\end{array}\right) .
$$

Покажем, что решение (55) описывает зарядово-сопряженное состояние. В $1+1$ измерениях при представлении (47) в качестве матрицы зарядового сопряжения (в области $x>0$ ) можно использовать равенство $C=\sigma_{3}$. Действительно, если решение уравнения (49) $\Psi_{n}$ с энергией $E_{n}$ найдено, то очевидно, что $\Psi_{n}^{c} \equiv \sigma_{3} \Psi_{n}^{*}$ также является решением, но с энергией $-E_{n}$. В частности, для решения с нулевой энергией (64) легко проверить, что $\sigma_{3} \Psi_{0}=\Psi_{0}$.

При квантовании энергетический спектр квантовых состояний в присутствии такой “нулевой моды” не должен изменяться, по крайней мере вплоть до порядка $\hbar$, однако, как показано в статье [15], другие свойства квантовых состояний, ассоциируемые с этими уровнями, оказываются измененными. Так, в присутствии зарядово-сопряженного состояния с нулевой энергией разложение операторов квантованного поля Дирака имеет вид

$$
\widehat{\Psi}(t, x)=c_{0} \Psi_{0}(x)+\sum_{n \geqslant 1}\left(c_{n} e^{-i E_{n} t} \Psi_{n}^{(+)}(x)+d_{n}^{+} e^{i E_{n} t} \Psi_{n}^{(-)}(x)\right),
$$

где $\Psi_{n}^{( \pm)}$- нормированные положительно- и отрицательно-частотные собственные функции, а $\pm E_{n}$ - собственные значения одночастичного гамильтониана Дирака во внешнем поле. Операторы $c_{0}, c_{n}$ и $d_{n}$ подчиняются стандартным антикоммутационным соотношениям $\left\{c_{n}, c_{k}^{+}\right\}=\delta_{n k}, n, k=0,1, \ldots,\left\{d_{n}, d_{k}^{+}\right\}=\delta_{n k}, n, k=1,2, \ldots$ Все остальные антикоммутаторы равны нулю.

Используя формулу (56), нетрудно установить, что имеются два независимых вырожденных состояния с нулевой энергией и фермионными зарядами $Q= \pm 1 / 2[15,16]$. Если бы можно было измерить электрический заряд фермионов этой двумерной модели в 
присутствии зарядово-сопряженного состояния с нулевой энергией, например поместив модель в слабое внешнее электромагнитное поле $A_{\mu}$, то электрический заряд фермионов, который обнаруживался бы полем $A_{\mu}$, был бы равен $e Q$, т.е. был бы полуцелым. В отсутствие внешнего скалярного поля фермионы обладали бы целым электрическим зарядом.

Благодарности. В заключение я хотел бы поблагодарить Чон Лин Хo (Choon-Lin Но) за ценные замечания.

\section{Список литературы}

[1] A. M. J. Schakel, G. W. Semenoff. Phys. Rev. Lett. 1991. V. 66. P. 2653.

[2] A. Neagu, A. M. J. Schakel. Phys. Rev. D. 1993. V. 48. P. 1785.

[3] $V . R$. Khalilov. Electrons in Strong Electromagnetic Fields: an Advanced Classical and Quantum Treatment. Amsterdam: Gordon \& Breach Sci. Publ., 1996.

[4] С. С. Геритейн, Я. Б. Зельдович. ЖЭТФ. 1969. Т. 57. С. 654

[5] J. Reinhardt, W. Greiner. Rep. Progr. Phys. 1977. V. 40. P. 219.

[6] J. Rafelski, L. P. Fulcher, A. Klein. Phys. Rep. C. 1978. V. 38. P. 227.

[7] M. Soffel, B. Müller, W. Greiner. Phys. Rep. C. 1982. V. 85. P. 51.

[8] В. С. Попов. Электродинамика сверхсильных кулоновских полей $(Z>137)$. Препринт ИТЭФ-169. М.: ИТЭФ, 1980.

[9] Я. Б. Зельдович, В. С. Попов. УФН. 1971. Т. 105. С. 403.

[10] А. Б. Мигдал. Фермионы и бозоны в сильных полях. М.: Наука, 1978.

[11] А. А. Гриб, С. Г. Мамаев, В. М. Мостепаненко. Вакуумные квантовые эффекты в сильных полях. М.: Энергоатомиздат, 1988.

[12] V. R. Khalilov, C. L. Ho. Mod. Phys. Lett. A. 1998. V. 13. P. 615; В. Р. Халилов. ТМФ. 1998. T. 116. № 2. C. 277.

[13] I. Pomeranchuk, Ya. Smorodinsky. J. Phys. USSR. 1945. V. 9. P. 97.

[14] И. С. Градитейн, И. М. Рыжик. Таблицы интегралов, сумм, рядов и произведений. М.: ГИФМЛ, 1963.

[15] R. Jackiw, C. Rebbi. Phys. Rev. D. 1976. V. 13. P. 3398.

[16] Р. Раджараман. Солитоны и инстантоны в квантовой теории поля. М.: Мир, 1985.

Поступила в редакцию 27.IV.1999 г. 